\title{
Comparative evaluation of different treatment schemes for aseptic meningoencephalitis in dogs of dwarf breeds
}

\author{
Eugene Marin ${ }^{1, *}$, Valery Ermolaev ${ }^{1}$, Oksana Marina $^{1}$, and Elena Rezaeva ${ }^{2}$ \\ ${ }^{1}$ Ulyanovsk State Agrarian University named after P.A. Stolypin, 432017 Ulyanovsk, Russia \\ ${ }^{2}$ AlternativaVet Veterinary Medicine Clinic, Samara, Russia
}

\begin{abstract}
The article presents the results of treatment of aseptic meningoencephalitis in dogs of dwarf breeds. Diagnostics included a thorough neurological examination: assessment of mental status, ability to perform diagnostic tests, proprioceptive and postural reaction, the consistency and symmetry of cranial reflexes, including of pupil response to light and response to the threat, the presence of involuntary movements and forced poses, the intensity of the muscle tone and tendon reflexes. If an animal is suspected of having a brain lesion, other possible causes of encephalopathy were excluded. For this purpose, a General blood test was performed (to detect signs of inflammation, anemia); a detailed biochemical blood test (excluding renal and hepatic encephalopathy, indirect signs of the presence of a portosystemic shunt); if a portosystemic shunt was suspected, a blood test for bile acids was performed; when excluding all other causes of damage to the central nervous system. Magnetic resonance imaging of the brain was performed with contrast with the use of the drug OmniScan. During the magnetic resonance imaging, multiple foci were detected in all parts of the brain, including in the thalamus and trunk; including in some animals, foci that have a tendency to decay and form a necrotic cavity. It was established that before the start of treatment, animals had a wobbly gait, convulsive signs, a comatose state in some dogs, and weak visual reflexes. As a result of studies of morphological and biochemical parameters of blood in dogs, it was found that all the data obtained were within the reference values and did not have significant significance in the diagnosis of aseptic meningoencephalitis. Against the background of etiotropic and symptomatic therapy, positive dynamics was observed in patients with aseptic meningoencephalitis.
\end{abstract}

\section{Introduction}

Aseptic meningoencephalitis in dogs is a group of noninfectious diseases that are characterized by inflammation of the brain. [1, p. 477] This is one of the most common brain pathologies common among dwarf dog breeds [2, p.50]. Age ranges of incidence are very wide [3, p. 155]. The average age is from 1 to 5 years, however, this pathology was recorded at the age of 7 months to 9 years. [4, p. 59].

With the growing popularity of dwarf dogs, the incidence of morbidity increases, owners increasingly turn to the clinic due to various neurological disorders in the pet, and thanks to modern diagnostic methods, it is possible to establish the cause of neurological deficiency in a timely manner and begin treatment $[5$, p. 470, 6, p. 982].

The study and implementation of new treatment regimens avoids the routine use of corticosteroid drugs, and, accordingly, the side effects caused by their prolonged use, such as hemorrhagic gastroenterocolitis, immunosuppression, secondary hyperadrenocorticism (iatrogenic cushing), chronic pancreatitis, and others [7, p. $255 ; 8$, p. $97 ; 9$, p. 723 ]. The aim of this work was to study the therapeutic efficacy of drug treatment of various forms of aseptic meningoencephalitis in dogs.

\section{Material and methods}

Diagnosis of aseptic meningoencephalitis was carried out according to the principle of an integrated approach:

- a thorough history taking (breed, age, nursery data, age, exact time of the beginning of the first clinical signs, conditions of keeping and feeding animals.);

- clinical examination: assessment of the general condition (condition of the coat and mucous membranes, presence or absence of signs of dehydration, measurement of blood pressure, measurement of blood glucose level);

- a thorough neurological examination: assessment of mental status, the ability to perform diagnostic tests, proprioceptive and postural reactions, consistency and symmetry of cranial reflexes, including the reaction of the pupil to light and reactions to the threat, the presence of involuntary movements and forced postures, the intensity of muscle tone and tendon reflexes.

\footnotetext{
Corresponding author: evgenimari@yandex.ru
} 
If the animal was suspected of having brain damage, other possible causes of encephalopathy were to be ruled out:

A general blood test (to detect signs of inflammation, anemia) is performed on an Abacus automated analyzer;

- An extensive biochemical blood test (excluding renal and hepatic encephalopathy, indirect signs of the presence of a portosystemic shunt) was performed on an automatic analyzer with Vetskan dry chemistry.- if there is a suspicion of a portosystemic shunt, a blood test is performed for bile acids. Serum samples are tested either on a clinical biochemical analyzer, or sent to the INVITRO laboratory.

- with the exclusion of all other causes of damage to the central nervous system, a magnetic resonance imaging examination of the brain with contrast is performed. For contrast, the drug Omniscan is used. When confirming the diagnosis using visual diagnostic methods, dogs are prescribed etiotropic and symptomatic therapy.

To assess the effectiveness of therapeutic regimens, we created two groups of animals that were treated according to different protocols.

Group No. 1: According to this protocol, animals received only methyl prednisolone succinate as an etiotropic therapy. An immunosuppressive dose of this drug was initially prescribed at the rate of $2 \mathrm{mg} / \mathrm{kg}$ every 12 hours, after 3 days the dose was reduced to 1.5 $\mathrm{mg} / \mathrm{kg}$ iv every 12 hours, after another 3 days to $1 \mathrm{mg} /$ $\mathrm{kg}$ iv 12 hours. The dosage of $1 \mathrm{mg} / \mathrm{kg}$ IV was maintained for 5 days. Then the dogs were transferred to the oral administration of methyl prednisolone at a dosage of $0.5 \mathrm{mg} / \mathrm{kg}$ and the intake was maintained for a long time. To stabilize the condition, a metipred in the form of a leophilisate was used in dosages from 2 to 1 $\mathrm{mg} / \mathrm{kg}$ every 12 hours. The drug was administered intravenously with a syringe dispenser at a rate of $100 \mathrm{ml}$ / hour, in order to minimize the possibility of side effects from the gastrointestinal tract. All animals of this group received iv mannitol in a dose of $1000 \mathrm{mg} / \mathrm{kg}$ once a

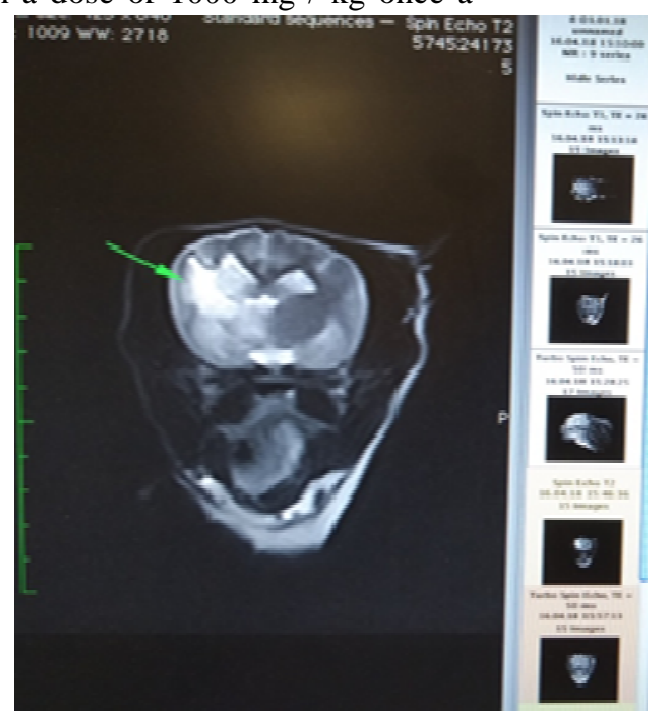

Fig. 1. On a series of MRI (tomography) in a T2 weighted image in the left frontal lobe, a focus with hyperintensive signaling characteristics is visualized that is not prone to decay. The T1 weighted image actively accumulates contrast material along the periphery. Group №1.

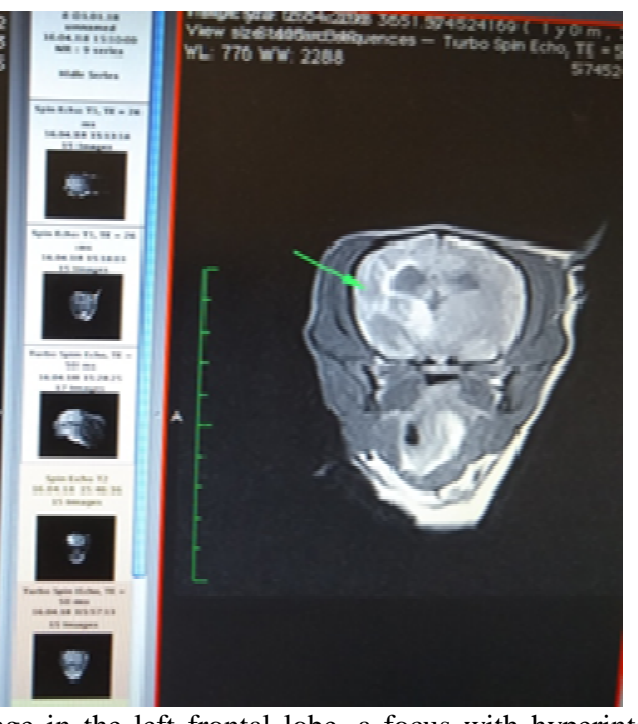

day for 3 days. In addition, all animals received omeprozole daily at a dose of $0.5 \mathrm{mg} / \mathrm{kg}$ in the morning 30 minutes before meals.

Group No. 2: According to the protocol, $4 \mathrm{mg} / \mathrm{kg}$ of Metipred was used once on the day of diagnosis. From the next day, dogs were given Metipred orally at a dosage of $0.5 \mathrm{mg} / \mathrm{kg}, 2$ times a day. From the second day of therapy, dogs were administered Cytosar in a dosage of $600 \mathrm{mg} / \mathrm{m}^{3}$. Infusion of the drug was carried out at a constant rate using a syringe dispenser for 10 hours. On the third day of therapy, a similar administration of the same dosages was carried out.

According to this scheme, taking Metipred in an antiinflammatory dosage lasted for 2 weeks, then it was canceled. Cytosar was used cyclically, repeated administration was prescribed 3 weeks after the first cycle, the drug was administered subcutaneously at a dosage of $300 \mathrm{mg} / \mathrm{m}^{3}$. Also in this group, dogs received Mannitol at a dosage of $1000 \mathrm{mg} / \mathrm{kg}$ intravenously for 15 ... 20 minutes for 3 days. For two weeks, dogs received omeprozole at a dosage of $2 \mathrm{mg} / \mathrm{kg} 30$ minutes phenobarbital at a dosage of $2.5 \mathrm{mg} / \mathrm{kg}$ every 12 hours.

\section{Results and Discussion}

The clinical picture in animals of group No. 1 was characterized as follows: at the initial intake, ataxia frolicking for two weeks was recorded. Dogs stagger when moving, cannot objectively assess the distance to furniture, stumbles upon objects. In addition, the dogs over the past day had 2 epileptic seizures of tonic-clonic seizures. On examination, the animals behave calmly, with free movement, there is a visible deviation from the axis to the left, there is no reaction to the threat on the left side, the pupil reaction is slowed down, mild horizontal nystagmus is noted. In some dogs, extensor hypertension was noted on the right side (Figures 1 and 2). before meals in the morning. One of the dogs received 


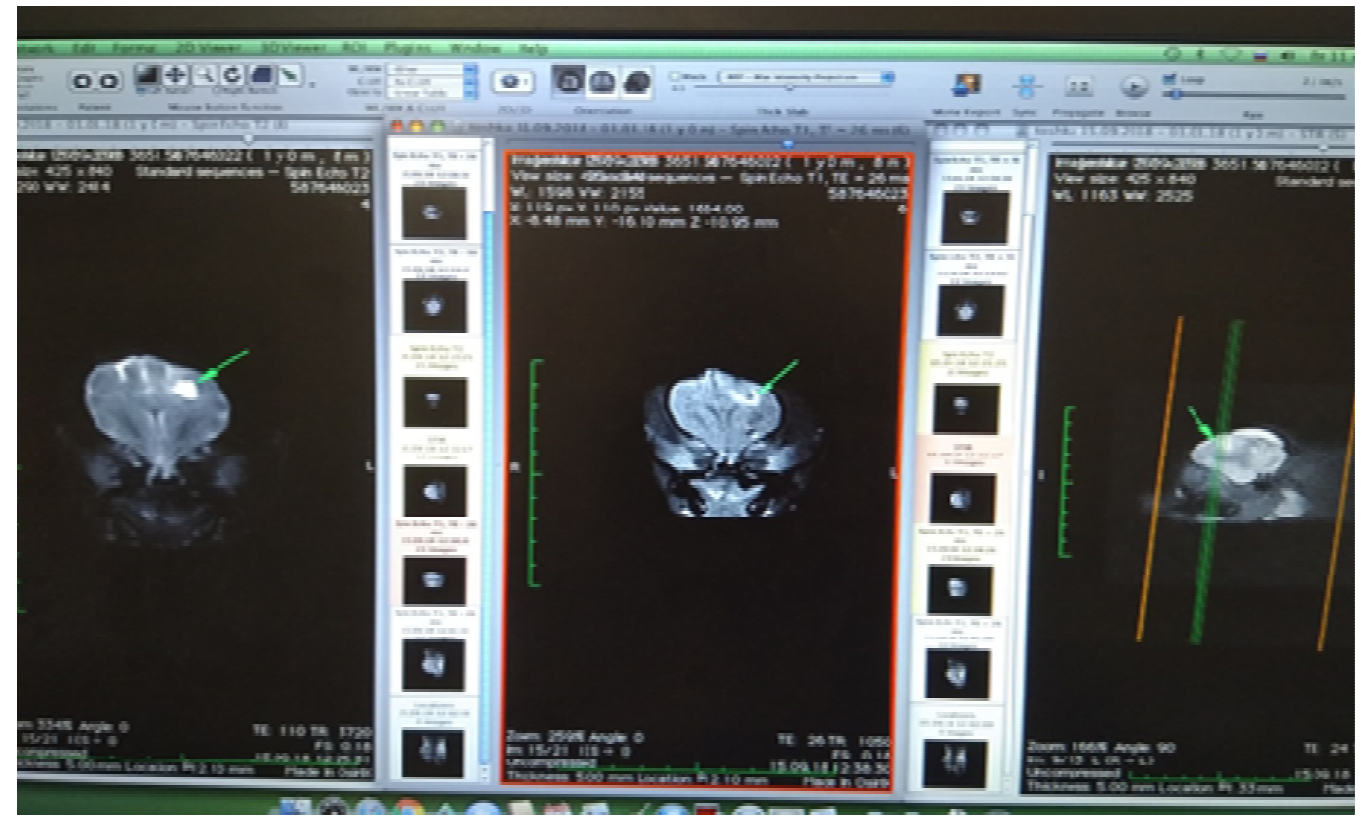

Fig. 2. A series of MRI (tomography) in a T2 weighted image visualizes a large focus in the temporal lobe of the right hemisphere, with hyperintensive signaling characteristics. In T1 mode, the tendency to necrotic decay is clearly expressed. Group 1
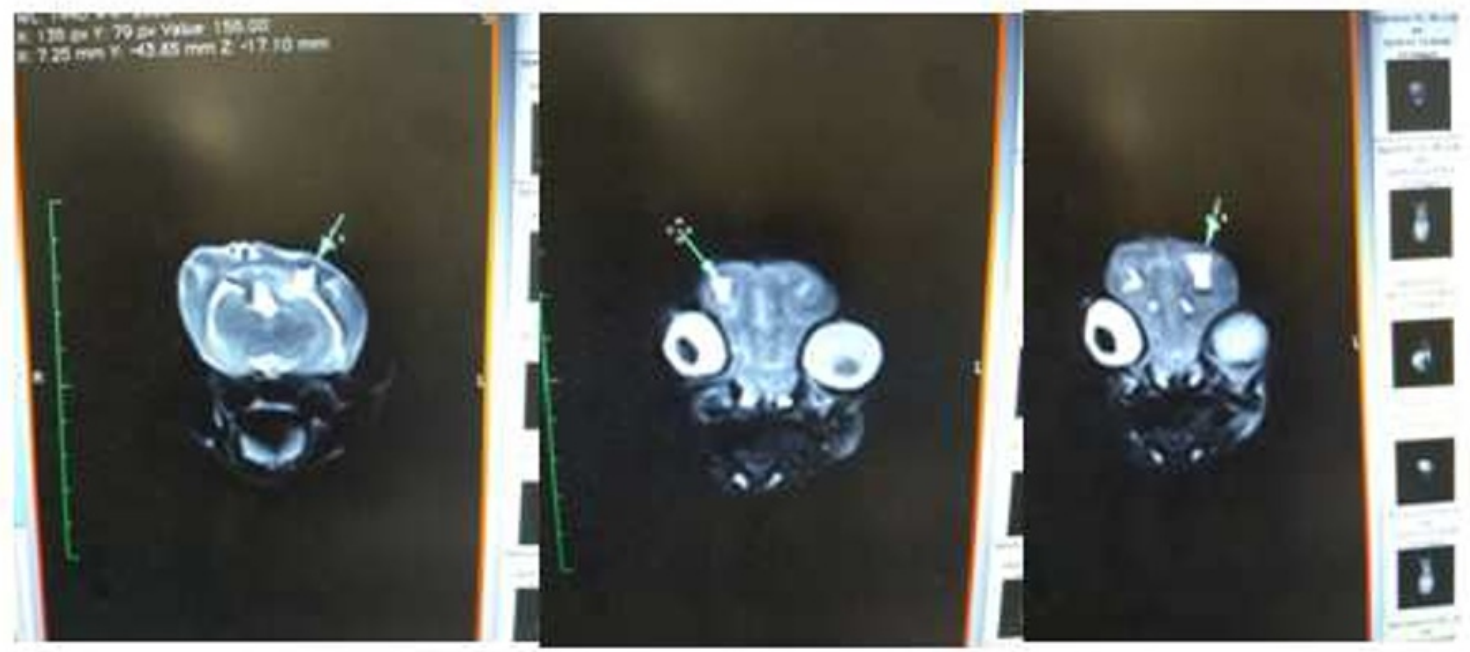

Fig. 3. On the MRI (tomography) series, multiple foci are visualized in a $T 2$ weighted image across all parts of the brain, including the thalamus and trunk. Group 2.

In animals in group No. 2, the following symptoms were recorded: general condition - deep stupor, there was no reaction to others. Forced position, lying down. Reflexes cannot be evaluated because of the state of stupor, close to a coma of the 1 st degree.

When examining other animals, bilateral mydriasis was recorded, lack of response to the threat on the right side, weakening of the pupillary reaction to the right eye. In addition, the animals have reduced appetite, there is aimless walking around the house, the animals hide, avoid contact with the owners. At the reception of tremor, dogs lower their heads down to the type of ventroflexion (Figure 3 and 4).

As a result of studies of morphological and biochemical blood parameters in dogs, it was found that all the results were within the reference values and did not have enough significance for the diagnosis of aseptic meningoencephalitis.

The condition of all animals from group No. 1 began to improve on average by $2 \ldots 4$ days of prescribed therapy. In dogs, there was a gradual smoothing of the clinical manifestations of the disease, signs of ataxia, and head tilts gradually passed. In patients with granulomatous meningoencephalitis, positive dynamics are more pronounced compared with patients with necrotizing forms of encephalitis.

On the 14th day of therapy in one patient at the neurological examination did not register any signs of neurological deficit. Some dogs on the 14th day of therapy because of neurological abnormalities had a proprioceptive deficiency on the right side and blindness in the right eye (patients did not recover completely in the future, but the quality of life was quite acceptable). 

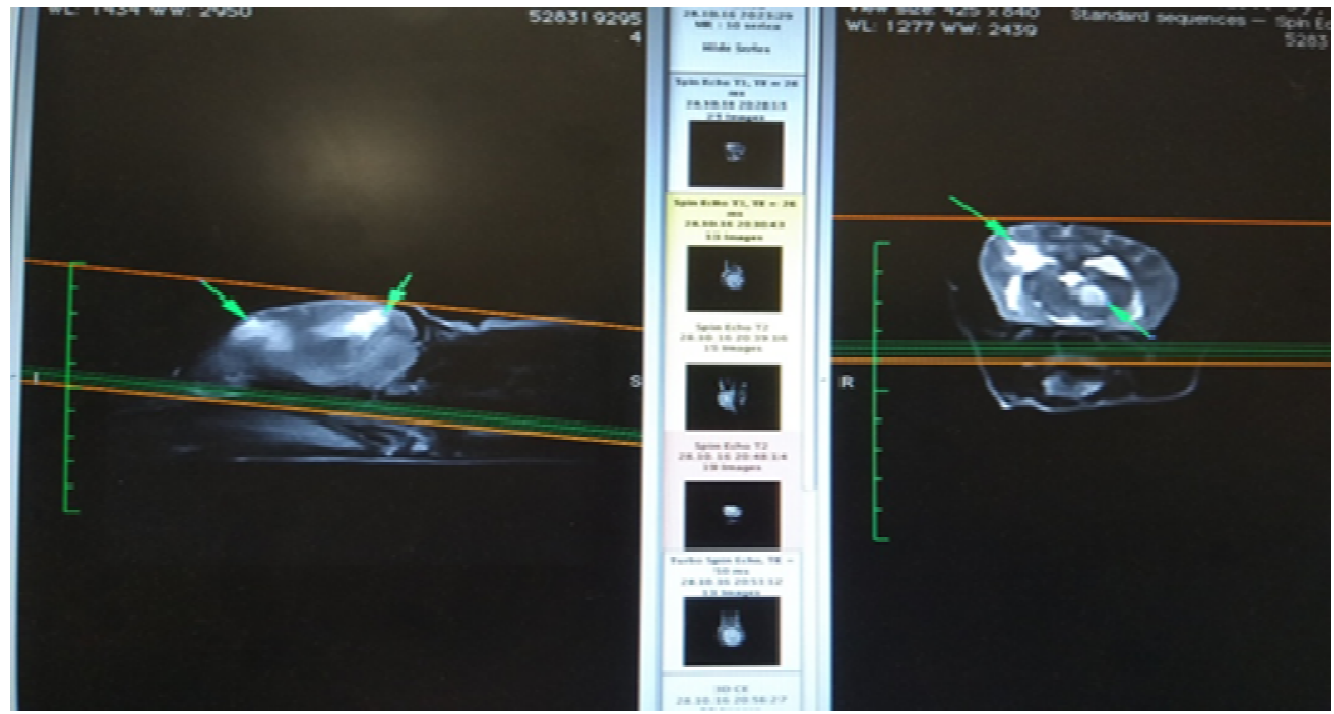

Fig. 4. On a series of MRI (tomography) in a T2 weighted image, foci in the frontal lobe and in the thalamus with hyperintensive signaling characteristics. In T1 weighted mode, the focus in the thalamus has a tendency to decay and form a necrotic cavity. Group 2 .

During the first three weeks, two patients developed signs of hemorrhagic gastroenteritis, which required the appointment of additional symptomatic therapy. One patient, after 2 months of methylprednisolone therapy, showed signs of iatrogenic cushing (deterioration in the quality of the coat, hyperkeratosis, apathy, decreased muscle tone of the abdominal wall). In addition, all patients in this group showed polyphagy, polydepsy and polyuria, which complicated the lives of the owners. The owners also noted that it is quite burdensome to give the dogs tablets every 12 hours on a daily basis.

In animals of group No. 2, the condition on day 2 of therapy was significantly more stable, and pronounced positive dynamics was observed. On day 14 of therapy, they were no different from healthy dogs. In all animals within 3 months of observation, there were no signs of damage to the gastrointestinal tract, the owners did not note polyuria and polydipsia, the dogs were active, and the quality of life was absolutely satisfactory.

It should be noted that in one animal of this group after 2 months of therapy, after the third cycle of Cytosar administration, inhibition of bone marrow function was observed, which was accompanied by the development of erythrocytopenia and anemia. The patient underwent a single transfusion of whole donated blood, after which the condition stabilized.

\section{Conclusion}

It should be noted that therapeutic measures in group No. 1 proved to be a fairly reliable solution to the problem of aseptic encephalitis, but was inconvenient in use and was accompanied by a large number of side effects. However, it should be noted that the choice of prescribing a particular treatment regimen should consist of a number of factors: the severity of the clinical picture, the degree of pathomorphological changes in the structure of the brain, the presence of concomitant diseases or a predisposition to them, the ability of the owners (the temporary possibility of owners, the ability to devote to their pet should be evaluated a large amount of time to comply with all recommendations).

\section{References}

1. F.C. Pellegrino, R.E. Sica, Canine electroencephalographic recording technique: findings in normal and epileptic dogs, Clinical Neurophysiology, 115, 477-487 (2004)

2. A.P. Kurdeko, A.A. Kozmidiadi, The state and prospects of the study of epilepsy in $\operatorname{dog}$ s, Scientific notes of the educational institution of the Vitebsk Order Badge of honor State Academy of Veterinary Medicine, 55(1), 50-53 (2019)

3. S. Platt, Small Animal Neurological Emergencies (2012) pp. 155-172

4. M. Podell, Antiepileptic drug therapy and monitoring, Top. Companion Anim. Med., 28, 59-66 (2013)

5. R.S. Fischer, Epileptic seizures and epilepsy: Definitions proposed by the International League Against Epilepsy (ILAE) and the International Bureau for Epilepsy (IBE), Epilepsia, 46, 470-472 (2005)

6. I.V. Nenashey, E.M. Marin, V.A. Ermolaev, Testing Of Intramuscular Administration Of Rometar On Pain Sensitivity And Clinical And Physiological Parameters In Rams Edilbaevskoy Breed, Research J. of Pharmaceutical, Biological and Chemical Sciences, 9(6), 982-986 (2018)

7. A. Pakozdy, P. Halaszand, A. Klang, Epilepsy in Cats. Theory and Practice, J. Vet. Intern. Med., 28(2), 255-263 (2014)

8. M. Podell, Manual of Canine and Feline Neurology BSAVA, Chapter 7, pp. 97-112 (2010)

9. J. D. Frost, Changes in epileptic spike configuration associated with attainment of seizure control, Annals of neurology, 20(6), 723-726 (1986) 